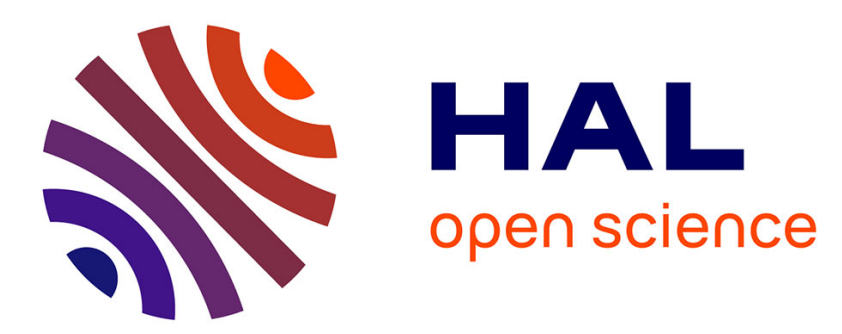

\title{
Impact of electric potential and magnetic drift on microtearing modes stability
}

\author{
M. Hamed, M. Muraglia, Yann Camenen, X. Garbet, O. Agullo
}

\section{To cite this version:}

M. Hamed, M. Muraglia, Yann Camenen, X. Garbet, O. Agullo. Impact of electric potential and magnetic drift on microtearing modes stability. Physics of Plasmas, 2019, 26 (9), pp.092506. 10.1063/1.5111701. hal-02408459

\section{HAL Id: hal-02408459 \\ https://hal.science/hal-02408459}

Submitted on 18 Nov 2020

HAL is a multi-disciplinary open access archive for the deposit and dissemination of scientific research documents, whether they are published or not. The documents may come from teaching and research institutions in France or abroad, or from public or private research centers.
L'archive ouverte pluridisciplinaire HAL, est destinée au dépôt et à la diffusion de documents scientifiques de niveau recherche, publiés ou non, émanant des établissements d'enseignement et de recherche français ou étrangers, des laboratoires publics ou privés. 


\title{
Impact of electric potential and magnetic drift on microtearing modes stability
}

\author{
M. Hamed, ${ }^{1,2}$ M. Muraglia, ${ }^{2}$ Y. Camenen, ${ }^{2}$ X. Garbet, ${ }^{1}$ and O. Agullo ${ }^{2}$ \\ 1) CEA, IRFM F-13108 St-Paul-Lez-Durance, France \\ 2) Aix-Marseille Université, CNRS, PIIM UMR 7345, Marseille, France
}

(Dated: October 10, 2019)

\begin{abstract}
The stability of a microtearing mode (MTM) as a function of collisonality is investigated by means of linear reduced model and numerical simulations using the gyrokinetic code GKW. The study is focused on the role of the electric potential and the magnetic drift, which are potential candidates to explain the destabilization of MTM observed at low collisionality in some recent gyrokinetic simulations. In the simulations, the magnetic drift and electric potential are found destabilizing in the presence of a finite collisionality. This destabilizating role is captured in the analytical calculation, which further highlights the requirement for a finite collisionality.
\end{abstract}

\section{INTRODUCTION}

Improvement of tokamak performance is possible thanks to the spontaneous onset of a high confinement mode (H-mode). The H-mode $\mathrm{e}^{1 / 2}$ is characterised by the formation of a narrow and insulating region inside the last closed flux surface, called pedestal. In this region, the radial transport is reduced and a steep pressure gradient is observed. The height and width of the pedestal strongly impacts the energy confinement time. Presently the "EPED" mode ${ }^{3 / 4}$, based on the stability of large and small scales MagnetoHydroDynamic (MHD) modes, is most commonly used to characterize the pedestal region. The EPED model has been extensively used and several numerical simulations using experimental data are in fair agreement with the observations. However, recent gyrokinetic simulations using the JET-ILW experimental equilibrium profils suggests that another class of instabilities, called microtearing modes (MTM), may also influence the pedestal height and width by generating a large electron heat transport leve ${ }^{5}$. MTM physics is not presently included in the EPED model. This microinstability is a possible candidate to explain the anomalous electron heat transport in tokamaks $6-8$. Microtearing modes (MTMs) are small scale tearing instabilities with a toroidal (n) and poloidal $(\mathrm{m})$ mode numbers larger than conventional tearing modes. MTM modifies the magnetic field line topology at the ion Larmor radius scale and leads to the formation of magnetic islands. The discovery of collisional MTM driven by electron temperature gradient is attributed to Hazeltine et al $! 9$, in 1975 and has been responsible for several developements of the linear stability theory. Past analytical work predicted a peaked growth rate of MTM at a finite value of collisionality and decrease down to a negative value in the collisionless regime. Hazeltine et al., proposed a kinetic description of a slab current sheet destabilized by an electron temperature gradient leading to a tearing instability in the collisional regime only. In 1977, Drake and Lee ${ }^{10}$, using a cylindrical geometry, studied in details the stability of MTMs driven by an electron temperature gradient in three regimes of collisionality, "collisionless", "semi-collisional" and "collisional". By means of analytical calculations they predicted unstable MTMs in the presence of collisions and stable MTMs in the collisionless regime. In 1980, Gladd et al ${ }^{11}$ established a new analytical calculation in slab geometry including the effect of the electric potential. The electric potential was found destabilizing in the presence of collisions. The role played by the trapped particles in MTMs destabilization has been investigated by Catto et al., in $1981^{12}$ and by Connor et al ${ }^{13}$ in 1990. A derivation of a dispersion relation in realistic tokamak geometry was formulated showing that trapped electrons are destabilizing in the presence of collisions. In 1989, Smolyakov ${ }^{14}$ showed that drift effects can give rise to small-scale magnetic islands called "drift magnetic islands". Then, in 1990, Garbet et al. ${ }^{15] 16}$ suggested analytically that MTM is unstable nonlinearly and generates electron heat transport. In summary, past analytical work predicted MTM stable in the collisionless regime and driven by the electron temperature gradient in a slab magnetised plasma. The analytical calculation was then improved in 2015 by Zocco et al. ${ }^{17}$. A novel hybrid fluid-kinetic model was developped, showing that the electric potential destabilizes MTMs in presence of collisions. The destabilization of MTM in the collisionless regime by the interchange effect was recently investigated by means of a fluid theory neglecting the electric potential 18 .

Clearly, the physical mechanisms at play in the MTMs destabilization in both the collisional and collisionless regime are not all well understood owing to the number of parameters (electric potential, interchange, trapped particles, magnetic drift,...) potentially involved. Futhermore, tokamaks operate 
at high temperature and therefore low collisionality at which MTM are not expected to be unstable. This claim caused a lessened interest in these micro instabilities as a relevant drive of turbulence. However, recent investigations using differents gyrokinetic codes predicted MTMs to be unstable in tokamak experimental conditions. MTM was found unstable in standard tokamak JET ${ }^{5202}$, ASDEX ${ }^{21 / 22]}$ and DIII-D ${ }^{23}$, in spherical tokamaks $6-24,28$ and in Reverse-field pinch 3031 . The existence of MTMs in tokamaks highlights the need to determine the role played by this micro-instability in differents collisionality regimes. Numerically, MTMs dominate at the top of the pedestal and can generate an electron heat transport. To better understand the mechanisms which can destabilize MTM, a reduced kinetic model must be extended to include the electric potential, the magnetic drift, the trapped particles, the interchange effect and the collisions.

In previous studies, a linear dispersion relation of a slab microtearing mode using a kinetic approach was established and compared with linear gyrokinetic simulations 33 . The linear stability of the collisionless MTMs predicted by the theory $9.14 \mid 16$ is found consistent with numerical simulations using the gyrokinetic code GKW ${ }^{32}$. MTMs are stable in collisionless regime. The purpose of this paper is to establish a kinetic reduced MHD model noted KRMHD model, including electric potential, magnetic drift, interchange effect and collisions to better understand their role in MTM destabilization. The effect of the trapped electrons is neglected in this reduced model. Then, the KRMHD model is compared with numerical simulations using GKW.

The paper is organized as follows. In Section If a dispersion relation of a current sheet model is derived including electric potential, magnetic drift and collisions in a kinetic framework. This KRMHD model is a derivation of a current sheet model by solving the Fokker-Planck and Maxwell equations. The FokkerPlanck equation, required to evaluate the current inside the resistive layer, is solved in ballooning representation. The full expression of the current inside the resistive layer is rather complex. This complex form can be simplified by using an effective magnetic drift. In section IA a dispersion relation including only the magnetic drift is found. It appears that the magnetic drift destabilizes MTMs only in conjunction with a finite collisionality 34 . In section IB the full electromagnetic problem is investigated. With both electric potential and magnetic drift, the evaluation of the current inside the resistive layer is obtained from a system of two equations linking the vector potential and the electric potential. This system of equations have been solved numerically using an eigenvalue code, called
"Solve_AP". In section [I] , a description of the gyrokinetic equations used in the GKW code is given. Stressing which terms need to be retained/neglected to solve equations similar to the KRMHD model. Physical mechanisms are added in GKW step by step to identify their effect on MTM stability.

\section{KINETIC MODEL OF MICROTEARING MODES}

The fundamental aspect of the physics behind the microtearing instability is the creation of a parallel current at a resonant flux surface $(q=m / n$, where $q$ is the safety factor). Current sheets are strongly localized around the resonant surface. Linear gyrokinetic simulations ${ }^{33}$ show that the mode structure is peaked at the low field side mid-plane and rotates in the electron diamagnetic direction. The width of the current layer, typically a few $\rho_{e}$, and the sensitivity of magnetic reconnection to dissipation make the numerical calculations of MTMs challenging: it requires a high numerical resolution and a very weak numerical dissipation, especially at low collisionality. The improvement of the analytical model is crucial, first to better understand the role played by the different physical parameters, but also because it is free from the sensitivity to numerical resolution and dissipation.

We consider a simple geometry of circular concentric magnetic surfaces where $r$ is the minor radius, $\varphi$ the toroidal angle and $\theta$ the straight field line poloidal angle. The ballooning representation ${ }^{19}$ appears as the appropriate tool to study MTM including the magnetic drift and the electric potential. The KRMHD model is derived in the "ballooning" space using the field aligned coordinates $(r, \alpha, \eta)$. Here, $\alpha=\varphi-q(r) \theta$ is a transverse coordinate and $\eta=\theta$ is the coordinate along the unperturbed field lines. Any perturbed field, for instance the vector potential $A_{\|}$, at given toroidal wave number $n$ and complex frequency $\omega$, is written:

$$
\begin{aligned}
& A_{\|}(r, \alpha, \eta, t)=\sum_{p=-\infty}^{\infty} \hat{A}_{\|}(\eta+2 p \pi) \\
& \exp \left\{i n \alpha+i n q(r)\left(\eta-\eta_{k}-2 p \pi\right)-i \omega t\right\}+\text { c.c. }
\end{aligned}
$$

Where $\mathrm{q}$ is the safety factor (near the rational surface $q=m / n$ where $\mathrm{m}$ and $\mathrm{n}$ are, respectively, the poloidal and the toroidal mode numbers) and $\eta_{k}$ is the ballooning angle. The evaluation of the perturbed current along the magnetic field line $J_{\|}=\sum_{\text {species }} e \int d^{3} v f_{n \omega} v_{\|}$ is obtained from the distribution function of each charged species whose evolution is given by the Fokker-Planck equation for each charged species, including the magnetic drift, electric potential fluctuations and collisions. The distribution function $f_{n \omega}$ for each species at given $n \omega$ is split in an unperturbed part 
$F_{M}$ taken as an unshifted Maxwellian of temperature $T_{e q}$, and a perturbed part $\hat{f}_{n \omega}\left(\eta, v_{\|}, \mu\right)$, where $v_{\|}$is the parallel velocity and $\mu$ the magnetic moment. The perturbed distribution function $\hat{f}_{n \omega}$ is itself written as the sum of an adiabatic part and a resonant part $\hat{f}_{n \omega}=-F_{M} \frac{\hat{h}_{n \omega}}{T_{e q}}+\bar{g}_{n \omega}$, where $\hat{h}_{n \omega}=e\left(\hat{\phi}-v_{\|} \hat{A}_{\|}\right)$is the perturbed Hamiltonian, $\hat{\phi}(\eta)$ and $\hat{A}_{\|}(\eta)$ are the $n, \omega$ Fourier components of the electric and vector potentials and $e$ is the algebraic charge. $\hat{A}_{\|}$and $\hat{\phi}$ determine the perturbed magnetic and electric fields: $\hat{\mathbf{B}}=\nabla \times\left(\hat{A}_{\|} \mathbf{b}\right)$ and $\hat{\mathbf{E}}=-\nabla \hat{\phi}-\frac{\partial \hat{A}_{\|}}{\partial t}$. The resonant part $\bar{g}_{n \omega}$, is solution of the following kinetic equation:

$$
\left(\omega-k_{\|} v_{\|}-\omega_{d}\right) \bar{g}_{n \omega}=\frac{F_{M}}{T_{e q}}\left(\omega-\omega^{*}\right) \mathcal{J} \hat{h}_{n \omega}+i \mathcal{C}\left(\bar{g}_{n \omega}\right)
$$

The complex frequency $\omega=\hat{\omega}_{r}+i \hat{\gamma}$ contains the mode frequency $\hat{\omega}_{r}$ and the mode growth rate $\hat{\gamma} \cdot \mathcal{J}$ is the gyro-average operator, $\mathcal{C}$ is a linearized Fokker-Planck electron-ion pitch-angle scattering collision operator and $\omega^{*}=\omega_{T}^{*}\left(\frac{1}{\eta_{e}}+\zeta^{2}-\frac{3}{2}\right)$ is the kinetic diamagnetic frequency with $\zeta=v / v_{T h e}$ and $\omega_{T}^{*}=\frac{k_{\theta} \rho_{i}}{2} \sqrt{\frac{m_{e}}{m_{i}}} \frac{v_{T h_{e}}}{R} \frac{R}{L_{T_{e}}}$ where $v_{T h e}=\sqrt{2 T_{e} / m_{e}}$ is the electron thermal velocity. The electron and ion mass and temperature are noted $m_{e}, m_{i}, T_{e}$ and $T_{i}$, respectively. In ballooning representation, $k_{\|}$must be understood as an operator which is a derivative along the magnetic field line $-i \frac{1}{q_{0} R_{0}} \frac{\partial}{\partial \eta}$. The magnetic drift frequency is defined as:

$$
\omega_{d}=\frac{n q_{0}}{r_{0}} \frac{m v_{\|}^{2}+\mu B_{0}}{e B_{0} R_{0}}\left(\cos \eta+s_{0}\left(\eta-\eta_{k}\right) \sin \eta\right)
$$

where $\eta_{k}$ is the ballooning angle and $s_{0}=\left.\frac{r d q}{q d r}\right|_{r=r_{0}}$ the magnetic shear at the reference resonant surface. Note that in ballooning representation $\left|\nabla_{\perp}\right|^{2}$ should be replaced by $k_{\perp}^{2}=k_{\theta}^{2}\left[1+s_{0}^{2}\left(\eta-\eta_{k}\right)^{2}\right]$, where $k_{\theta}=-\frac{n q_{0}}{r_{0}}$ is the reference poloidal wave number. Finally it is convenient to replace the distribution function $\bar{g}_{n \omega}$ by $\hat{g}_{n \omega}=\bar{g}_{n \omega}-\frac{F_{M}}{T_{e q}} e \frac{\omega-\omega^{*}}{\omega} \mathcal{J} \hat{\phi}$. The linear dispersion equation for MTM including both magnetic drift and electric potential can be obtained by solving the Ampère law and Poisson equation written in a variational form:

$$
\mathcal{L}=-\frac{1}{\mu_{0}} \int d^{3} \mathbf{x}\left|\nabla_{\perp} A_{\|}\right|^{2}+\int d^{3} \mathbf{x}\left(J_{\|} A_{\|}^{*}-\rho \phi^{*}\right)
$$

where $\rho$ is the charge density and the complex conjugate of the electric potential and the vector potential are noted respectively $\phi^{*}$ and $A_{\|}^{*}$. The extremalisation of the functional with any variation of $\phi^{*}$ and $A_{\|}^{*}$ is equivalent to the linearized Poisson and Ampère equations. One important property of $\mathcal{L}$ is that it vanishes when $\phi$ and $A_{\|}$match the solution of Maxwell equations. It turns out that the Hamiltonian formulation is not the most suitable one to get close to the traditional resistive MHD formulation. Indeed, one would like to involve the parallel electric field rather than a perturbed Hamiltonian $\hat{h}_{n \omega}$. This is done via the following transformation:

$$
\frac{\hat{h}_{n \omega}}{e}=\frac{\omega-k_{\|} v_{\|}-\omega_{d}}{\omega} \hat{\phi}+\frac{\omega_{d}}{\omega} \hat{\phi}-v_{\|} \hat{\mathcal{E}}_{\|}
$$

Where $\hat{\mathcal{E}}_{\|}=\hat{A}_{\|}-\frac{k_{\|}}{\omega} \hat{\phi}$ is proportional to the parallel electric field. Replacing the expression of the perturbed current in the functional we obtain the following expression:

$$
\begin{aligned}
\mathcal{L} & =-\frac{1}{\mu_{0}} \int d^{3} \mathbf{x}\left|\nabla_{\perp} \hat{A}_{\|}\right|^{2} \\
& +\int d \tau \frac{F_{M} e^{2}}{T_{i, e q}} \frac{\omega-\omega_{i}^{*}}{\omega}\left(1-\mathcal{J}^{2}\right)|\hat{\phi}|^{2} \\
& +\sum_{\text {species }} \int d \tau \frac{F_{M} e^{2}}{T_{\text {eq }}} \frac{\omega^{*} \omega_{d}}{\omega^{2}}|\mathcal{J} \hat{\phi}|^{2}+\sum_{\text {species }} \mathcal{L}_{\text {res }}
\end{aligned}
$$

where

$$
\mathcal{L}_{\text {res }}=-e \int d \tau \hat{g}_{n \omega} \mathcal{J}\left(\frac{\omega_{d}}{\omega} \hat{\phi}-v_{\|} \hat{\mathcal{E}}_{\|}\right)^{*}
$$

where $d \tau=d^{3} \mathbf{x} d^{3} \mathbf{p}$ is the volume element in the ballooning phase space. The first term represents the magnetic energy, and gives rise to the field part of the Ampère equation. The second term is the polarization term (including diamagnetic corrections): it results from both ion and electron contributions. The third term is the interchange drive responsible for the ballooning instability. The last term is associated with the resonant response of ions and electrons. The latter is of particular interest. Ions are considered collisionless and the ion resonant contribution is neglected. An electron-ion pitch-angle scattering collision operator is used in the Eq.(2):

$$
\mathcal{C}\left(\hat{g}_{n \omega}\right)=\frac{1}{2} v_{e i}(v) \frac{\partial}{\partial \xi}\left(1-\xi^{2}\right) \frac{\partial \hat{g}_{n \omega}}{\partial \xi} .
$$

Where $v_{e i}(v)$ is the electron-ion collisional frequency and $\xi=\frac{v_{\|}}{v}$ is the pitch-angle variable (which varies between -1 and 1 and $v$ the velocity modulus). It is then convenient to expand the perturbed distribution function over a basis of Legendre polynomials restricted to the first two components. Then, the distribution function becomes a solution of a differential equation that is solved using a WKB approximation ${ }^{35}$. More details about this calculation are given in 34 Eq. (6) is the exact solution of the problem including electric potential, magnetic drift, interchange effect and collisions. This solution is not tractable. To simplify it, an effective curvature magnetic drift is employed in the following. 


\section{A. Effect of Magnetic drift}

\section{Asymptotic matching in ballooning space}

In this part, we focus on the magnetic drift and neglect the electric potential. Outside the resistive layer (currentless region), $A_{\|}$decays as $A_{\|}(0) e^{-\left|k_{\theta} x\right|}$, where $\mathrm{x}$ is the radial distance to the resonant surface of the mode. Note that the slab current sheet is very thin and localized around the resonant surface:

$$
\delta_{J}=\frac{L s}{L_{T e}} \rho_{e} \sqrt{\frac{v_{e i}}{\omega^{*}}}<<\frac{1}{k_{\theta}}
$$

Where $L_{s}$ is magnetic shear length $L_{s}=q R / \hat{s}, L_{T_{e}}$ is the electron temperature gradient length scale and $\rho_{e}$ is the electron Larmor radius. Inside the layer where a current develops, $A_{\|}$is assumed to vary slowly consistently with a "constant $A_{\|}$" approximation $\left(A_{\|}\right.$is constant in the current layer) 3 . The matching of the internal and external solutions in physical space is presented in 3437 . Here the same procedure is translated in the ballooning space. The external mode away from the resistive layer in physical space becomes a localized solution, near $\eta=0$ in ballooning space.

$$
\hat{A}_{\|}(\eta)=-\mu_{0} \frac{\hat{J}_{\|}(0)}{\left[1+s_{0}^{2} \eta^{2}\right] k_{\theta}^{2}}=\hat{A}_{\|}(0) \frac{1}{1+s_{0}^{2} \eta^{2}}
$$

Where $\nabla_{\perp}^{2} \hat{A}_{\|}(\eta)$ is replaced by $k_{\perp}^{2} \hat{A}_{\|}(\eta)=k_{\theta}^{2}[1+$ $\left.s_{0}^{2}\left(\eta-\eta_{k}\right)^{2}\right]$ in the Ampère equation. Eq. 10 yields a localization of $A_{\|}$around $\eta=0$ (low field side midplane) comparable to that obtained in the simulations, see Fig. (4). Then, the internal solution $\hat{A}_{\|}(x)$ which is constant in the physical space becomes a function $\hat{A}_{\|}(\eta)$ in the ballooning space. Note that, for circular flux surfaces and small aspect ration $\epsilon=r / R<<1$, with $r$ and $R$ the minor and major radius of the surface, respectively the coordinate along the magnetic field lines used in GKW becomes $s=\eta / 2 \pi \underline{39}$. This asymptotic matching gives the new following expression of the functional:

$$
\mathcal{L}=\mathcal{L}_{\text {mag }}+\mathcal{L}_{\text {res }}
$$

where

$$
\mathcal{L}_{\text {mag }}=-\frac{1}{\mu_{0}} \int_{-\infty}^{+\infty} \frac{d \eta}{2 \pi} k_{\theta}^{2}\left[1+s_{0}^{2} \eta^{2}\right]\left|\hat{A}_{\|}(\eta)\right|^{2}
$$

and $\mathcal{L}_{\text {res }}$ is given by Eq. (7) with $\hat{\mathcal{E}}_{\|}=\hat{A}_{\|}$, when the electric potential is neglected. We restrict the analysis to a zero ballooning angle $\eta_{k}=0$, localized mode. Plugging Eq. (10) into the functional $\mathcal{L}_{\text {mag }}$ provides the following form of $\mathcal{L}_{\text {mag }}$

$$
\mathcal{L}_{\text {mag }}=-\frac{2\left|k_{\theta}\right|}{\mu_{0}|d|}\left|A_{\|}(0)\right|^{2}
$$

Where $d$ is the distance between resonant surfaces. All calculations done, the resonant term becomes:

$$
\begin{aligned}
\mathcal{L}_{\text {res }} & =i \frac{\pi}{\sqrt{3}} q R_{0}\left|A_{\|}(0)\right|^{2} \\
& \times \int_{0}^{+\infty} d v 4 \pi v^{3} \frac{F_{M e^{2}}}{T_{e q}} \frac{\omega-\omega^{*}}{\left(1+i \frac{v_{e i}(v)}{\omega-\omega_{d}(v, 0)}\right)^{1 / 2}}
\end{aligned}
$$

Hence the total functional $\mathcal{L}=\mathcal{L}_{\text {mag }}+\mathcal{L}_{\text {res }}$ becomes

$$
\begin{aligned}
\mathcal{L} & =-\frac{2\left|k_{\theta}\right|}{\mu_{0}|d|}\left|A_{\|}(0)\right|^{2} \\
& +i \frac{\pi}{\sqrt{3}} q R_{0}\left|A_{\|}(0)\right|^{2} \\
& \times \int_{0}^{+\infty} d v 4 \pi v^{3} \frac{F_{M} e^{2}}{T_{e q}} \frac{\omega-\omega^{*}}{\left(1+i \frac{v_{e i}(v)}{\omega-\omega_{d}(v, 0)}\right)^{1 / 2}}
\end{aligned}
$$

Where $\omega_{d}(v, \eta)=\omega_{d}(v, 0)$.

\section{Effect of magnetic drift on microtearing modes stability}

Starting from Eq. 15), a dispersion relation including the magnetic drift and collisions is formulated. It is convenient to normalize all frequencies to the electron temperature diamagnetic frequency $\omega_{T e}^{*}=\frac{k_{\theta} T_{e, e q}}{e B_{0} L_{T e}}$ and all velocities are normalized to the electron thermal velocity $v_{T e}=\sqrt{\frac{2 T_{e, e q}}{m_{e}}}$. Hence, $\Omega=\frac{\omega}{\omega_{T e}^{*}}, \Omega_{d}(v, \eta)=$ $\frac{\omega_{d}(v, \eta)}{\omega_{T e}^{*}}, \Omega^{*}=\frac{\omega^{*}}{\omega_{T e}^{*}}$ and $\bar{v}_{e i}=\frac{v_{e i, t h}}{\omega_{T e}^{*}}$, where $v_{e i, t h}$ is the thermal collision frequency. The condition $\mathcal{L}=0$ then reads

$$
\frac{1}{\hat{\beta}}=8 i \sqrt{\frac{\pi}{3}} \int_{0}^{+\infty} d \xi \xi^{\frac{9}{2}} e^{-\xi^{2}} \frac{\Omega-\Omega^{*}(\xi)}{\left[\xi^{3}+i \frac{\bar{v}_{e i}}{\Omega-\Omega_{d} \xi^{2}}\right]^{1 / 2}}
$$

where $\hat{\beta}=\frac{2 \mu_{0} N_{e q} T_{e, e q}}{B^{2}} \frac{q_{0} R_{0}}{s_{0} L_{T e}} \frac{1}{k_{\theta} \rho_{e}}, \rho_{e}=\frac{m_{e} v_{T e}}{e B_{0}}, \Omega^{*}(\xi)=$ $\frac{1}{\eta_{e}}+\xi^{2}-\frac{3}{2}, \eta_{e}=\frac{L_{n e}}{L_{T e}}$ and $\Omega_{d}=\frac{L_{T e}}{R_{0}} \frac{4}{3}$. In the absence of magnetic drift $\Omega_{d}=0$, the previous result obtained for a slab collisional tearing mode is qualitatively recovered 33 . The magnetic drift is found to be destabilising, but only in conjunction with a finite collisionality 34 . The use of an effective magnetic drift is somewhat justified by the localization of the mode around the low-field-side midplane, Fig. 4. A comparison between the analytical calculation Eq. 16 and numerical simulations will be presented in section II The logical next step to understand the MTMs destabilization mechanisms is to add the electric potential in the calculation. 


\section{B. Effect of the electric potential in MTM destabilization}

We now go back to the full electromagnetic problem. In the reduced model, the Fokker-Planck equation is averaged over the cyclotronic motion (gyroaverage). $\mathcal{J}$ is the gyroaverage operator which is defined as the Fourier transform of the Bessel function of zero-th order. For $k_{\perp} \rho_{i}<<1$, the Bessel function is approximated and replaced by $1-\mathcal{J}^{2}=\frac{1}{2} k_{\perp}^{2} \rho_{i}^{2}$ for ions and by 1 for electrons, $\frac{\rho_{e}}{\rho_{i}}=\sqrt{\frac{m_{e}}{m_{i}}}<<1$. In the resistive layer, $k_{\perp} \simeq k_{r} \simeq 1 / \delta_{e}, k_{\perp}$ and $k_{r}$ are the perpendicular and radial wave number and $\frac{\delta_{e}}{\rho_{i}}=\frac{\rho_{e}}{\rho_{i}} \frac{L_{s}}{L_{T e}}$ is the collisionless layer width. The KRMHD model is valid for the following condition, $\delta_{e}>>\rho_{i}$. In the opposite limit $\left(k_{\perp} \rho_{i} \rightarrow+\infty\right)$, this approximation is not valid. Let us start from Eq.(6). The ion polarization functional then becomes:

$$
\begin{aligned}
\int d \tau \frac{F_{M} e^{2}}{T_{i, e q}} \frac{\Omega-\Omega_{i}^{*}}{\Omega}\left(1-\mathcal{J}^{2}\right)|\hat{\phi}|^{2}= \\
\quad \int d^{3} \mathbf{x} \frac{N_{e q} m_{i}}{B_{0}^{2}} \frac{\Omega-\Omega_{p i}^{*}}{\Omega}\left|\nabla_{\perp} \hat{\phi}\right|^{2}
\end{aligned}
$$

where $\Omega_{p i}^{*}=\frac{\omega_{p i}^{*}}{\omega_{T e}^{*}}$ with $\omega_{p i}^{*}=-\frac{k_{\theta} T_{i, e q}}{e_{p} B_{0} L_{p i}}, L_{p i}$ is the ion pressure gradient length. The final form of the functional then becomes

$$
\begin{aligned}
\mathcal{L} & =-\frac{1}{\mu_{0}} \int d^{3} \mathbf{x}\left|\nabla_{\perp} \hat{A}_{\|}\right|^{2} \\
& +\int d^{3} \mathbf{x} \frac{N_{e q} m_{i}}{B_{0}^{2}} \frac{\Omega-\Omega_{p i}^{*}}{\Omega}\left|\nabla_{\perp} \hat{\phi}\right|^{2} \\
& +\sum_{\text {species }} \int d^{3} \mathbf{x} \frac{N_{e q} m_{i}}{B_{0}^{2}} \frac{\Omega^{*} \Omega_{d}}{\Omega^{2}}|\mathcal{J} \hat{\phi}|^{2} \\
& +\mathcal{L}_{\text {res }}
\end{aligned}
$$

The complex form of the functional Eq.(18) can be simplified by assuming as in section IA an effective magnetic drift.

$$
\mathcal{L}_{\text {res }}=\frac{1}{\mu_{0} d_{e}^{2}} \int_{-\infty}^{\infty} \frac{d x}{|d|} \sigma(x)\left|\hat{A}_{\|}-\frac{k_{\|}}{\omega} \hat{\phi}\right|^{2}
$$

Where $d_{e}^{2}=\frac{c}{\omega_{p e}}$ is the electron skin depth $\left(\omega_{p e}^{2}=\right.$ $N_{e q} e^{2} / m_{e} \epsilon_{0}, \omega_{p e}$ is the electron plasma frequency). The dimensionless "conductivity" $\sigma$ is defined as

$$
\sigma(\rho)=-\frac{2}{3} \int_{0}^{+\infty} d \zeta F_{M} \zeta^{2} \frac{\Omega-\Omega^{*}(\zeta)}{\Omega-\Omega_{d} \zeta^{2}+i \frac{\hat{v_{e i}}}{\zeta^{3}}-\frac{1}{3} \frac{\rho^{2} \zeta^{2}}{\Omega-\Omega_{d} \zeta^{2}}}
$$

where $\Omega_{d}$ is an effective magnetic frequency calculated at $\eta=0$ and $\rho=x / \delta_{e}$ is the radial coordinate. The extremalization of the functional in the physical space provides a set of two equations

$$
\begin{aligned}
\nabla_{\perp}^{2} \hat{\psi}+\beta^{*} \sigma(\rho, \zeta)\left(\hat{\psi}-\frac{\rho}{\Omega} \hat{\phi}\right) & =0 \\
\nabla_{\perp}^{2} \hat{\phi}+\mu_{e} \frac{\rho}{\Omega} \sigma(\rho, \zeta)\left(\hat{\psi}-\frac{\rho}{\Omega} \hat{\phi}\right)-C_{i n t} \hat{\phi} & =0
\end{aligned}
$$

Where $\hat{\psi}=v_{\text {the }} \hat{A}_{\|}$and

$$
\mu_{e}(\Omega)=\frac{m_{e}}{m_{i}} \frac{L_{s}^{2}}{L_{T e}^{2}} \frac{1}{\frac{1}{\Omega}\left(\frac{1}{\eta_{e}}+1\right)+1} \text { and } \beta^{*}=\left(\frac{L_{s}}{L_{T e}}\right)^{2} \beta_{e}
$$

The quantities $\left(\beta^{*}, \mu_{e}\right)$ are defined to have compact equations. $C_{i n t}$ is a coefficient associated with the interchange term:

$$
C_{\text {int }}=\frac{\left(1+\frac{1}{\eta_{e}}\right)}{1+\frac{1}{\Omega}\left(1+\frac{1}{\eta_{e}}\right)} \frac{1}{\Omega^{2}}\left(\frac{L_{s}}{L_{T}}\right)^{2} \frac{m_{e}}{m_{i}} \frac{2 L_{T}}{R}
$$

This system is nothing else than the equations of reduced MHD where the Spitzer ${ }^{38}$ conductivity is replaced by the kinetic conductivity $\sigma(\rho, \zeta)^{30}$. We call it kinetic reduced MHD model (KRMHD). To solve Eqs. 21/22) this system of equations, is written in a matrix form. Using finite differences $\hat{A}_{\|}$is assuming to decay at large $|x|$ as $\hat{A}_{\|} \sim \exp \left(-\left|k_{\theta}\right||x|\right)$. The matching with the external solution is represented by the $\Delta^{\prime}$ parameter, which defined for a microtearing mode by $\Delta^{\prime}=-2\left|k_{\theta}\right|$. The asymptotic matching allows fixing the boundary conditions. When $x=0$, the boundary conditions are: $\left.\frac{d \hat{A}_{\|}}{d x}\right|_{x=0}=0$ and $\hat{\phi}(0)=0$. When $|x| \rightarrow+\infty$, the conditions are: $|\hat{\phi}| \rightarrow 0$ and $\left|\hat{A}_{\|}\right| \rightarrow \exp \left(-\left|k_{\theta}\right||x|\right)$. Eqs. $21 \mid 22$ are solved far away from the current layer. These equations are then implemented in an eigenvalue code, called "Solve_AP". The results obtained from "Solve_AP" are directly compared with gyrokinetic simulations using a version of GKW close to the theoretical model. A reasonable agreement is observed in the long wavelength limit, $\delta_{e}>>\rho_{i}$. Fixing revelant physical parameters (Table III) to be in this limit, we seek the growth rate and the frequency of the mode solution of this system of equations. This comparison is presented in section III

\section{NUMERICAL INVESTIGATION OF THE STABILITY OF MTMS}

All the results presented in this part use the local version of GKW. The gyrokinetic Fokker-Planck equation of the perturbed distribution function $\hat{f}_{g y}$ is solved in the electromagnetic case with a perturbed electric potential $\hat{\phi}$ and a pertubed vector potential $\hat{A}_{\|}$ in the flux-tube approximation. To be closer to the 
assumption of the analytical calculation of section I, a linearized collision operator with pitch- angle scattering only is used. Non-linearities, particle trapping, neoclassical transport and magnetic field compression are switched off. The gyrokinetic equation solved in this section can be written in the form:

$$
\frac{\partial g}{\partial t}=I+I I+V+V I I+V I I I
$$

where

$$
\begin{aligned}
& I=-v_{\|} \mathbf{b} \cdot \nabla \hat{f}_{g y} \\
& I I=-v_{d} \cdot \nabla \hat{f}_{g y} \\
& V=-v_{\chi} \cdot \nabla F_{M} \\
& V I I=\frac{Z e}{T} v_{\|} \cdot \nabla \mathcal{J} \phi F_{M} \\
& V I I I=-\frac{Z e}{T} v_{d} \cdot \nabla \mathcal{J} \phi F_{M}
\end{aligned}
$$

The notations of these terms follow the notations used in the GKW user guide ${ }^{39}$. I is the free streaming motion along the field line, II is the magnetic drift in the perturbed distribution function, $\mathrm{V}$ is the perturbed drift into the background distribution, VII is the parallel Landau damping term, VIII is the perpendicular Landau damping. In the terms written above $v_{\|} \mathbf{b}$ is the parallel motion along the unperturbed field, $v_{d}$ the drift motion due to the inhomogeneous field and $v_{\chi}$ is a combinaison of the perturbed $\mathbf{E} \times \mathbf{B}$ velocity $\left(\mathbf{v}_{E}=\mathbf{b} \times \nabla \mathcal{J} \phi / \mathbf{B}\right)$ and the parallel motion along the perturbed field line $\left(\mathbf{v}_{\delta \mathbf{B}}=-\mathbf{b} \times \nabla v_{\|} \mathcal{J} A_{\|} / B\right)$ then $\mathbf{v}_{\chi}=\mathbf{v}_{E}+\mathbf{v}_{\delta \mathbf{B}} . F_{M}$ is a Maxwellian and $\hat{g}_{g y}=\hat{f}_{g y}+\frac{Z e}{T} v_{\|} \cdot \nabla \mathcal{J} A_{\|} F_{M}$. The revelant physical parameters used in the simulations are presented in Table I. MTMs are sensitive to $\beta$ (the

Table I: Revelant input parameters used for the theoretical calculations and the linear simulations with GKW.

\begin{tabular}{cccccccc}
\hline$q$ & $\mathrm{~s}$ & $\beta(\%)$ & $R / L_{T e}$ & $R / L_{T i}$ & $R / L_{n e}$ & $\epsilon$ & $k_{\theta} \rho_{i}$ \\
\hline 1.34 & 1.08 & 1.55 & 8 & 0 & 0.26 & 0.22 & 0.3 \\
\hline
\end{tabular}

ratio of plasma kinetic to magnetic pressure) and to the electron temperature gradient $R / L_{T e}$ 9110133. Their values are chosen to have a sufficiently high MTM growth rate at high collisionality. As explained in the introduction the numerical study of MTM is challenging. MTMs are localized around the resonant surface. The current sheet is very thin, which implies having a high numerical resolution and a weak numerical dissipation, in particular at low collisionality. At low collision frequency the artificial numerical dissipation implemented in the code to ensure the stability of the numerical schemes can play the role of collisions leading to a destabilization of MTM in this regime.
Table II: Numerical resolution using in gyrokinetic simulations.

\begin{tabular}{cccc}
\hline $\mathbf{N}_{\mu}$ & $\mathbf{N} v_{\|}$ & $\mathbf{N}_{s}$ & $\mathbf{N}_{p o l}$ \\
\hline 16 & 60 & 79 & 99 \\
\hline
\end{tabular}

The numerical resolution used for the numerical simulations is presented in Table II The number of points per poloidal turns $\mathrm{N}_{\mathrm{s}}$ and the number of poloidal turns $\mathrm{N}_{\text {pol }}$ (an equivalently connected radial modes) have been adjusted to enable an accurate description of the mode. Note that for ITG modes, $N_{s}=24$ and $\mathrm{N}_{\text {pol }}=5$ is usually sufficient. $N_{\mu}$ is the number of magnetic moment grid points, $\mathrm{N}_{\mathrm{v}_{\|}}$is the number of parallel velocity grid points.

\section{NUMERICAL INVESTIGATION OF THE ROLE PLAYED BY THE ELECTRIC POTENTIAL AND MAGNETIC DRIFT}

\section{A. MTMs unstable in presence of collisions}

The role played by the electric potential and the magnetic drift is investigated numerically. The electric potential and magnetic drift are switched on step by step in the GKW to better understand their role in the MTM destablisation. Fig. 1 illustrates the role played by the magnetic drift and the electric potential. Without electric potential and without magnetic drift (black square), it appears that MTMs are stable at both low and high collisionality. For intermediate collisionality, typical of value in the pedestal region, MTMs are unstable, destabilized by the electron temperature gradient. A fair agreement between analytical calculation and numerical simulations was found, details

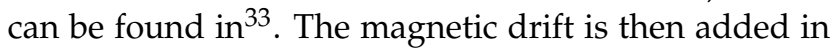
the code (green plus sign curve). Without the electric potential, the magnetic drift destabilizes MTMs in the intermediate collisionality. The role played by the electric potential is now investigated. The electric potential has been added in GKW and the magnetic drift has been switched on or off (the blue triangle and the red cross curve in the Fig. 1 respectively. It appears, without magnetic drift and with electric potential, another branch with opposite parity appears at high collisionality (purple triangle). These modes are giant electrons tails 40 . The growth rate definitely vanishes at low collisionality, as shown in Fig. 1 whereas the growth rate of MTMs increases then rolls over and decreases in the strong collisional regime. Finally, with magnetic drift and the electric potential (red cross) MTMs are more unstable but still stabilized at very low electronion collisions. For this set of parameters (Table $\llbracket$, the electric potential and magnetic drift destabilize MTM 


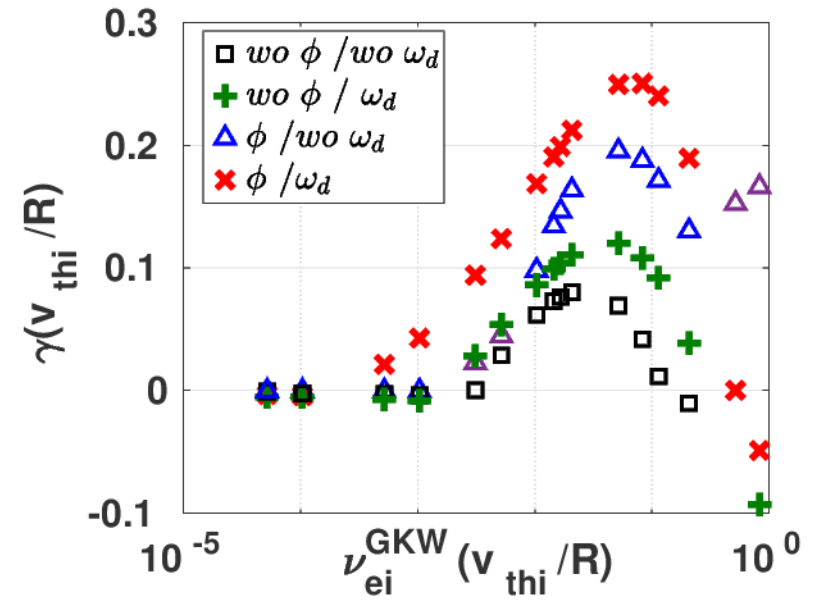

Figure 1: Growth rate of the microtearing mode as function of the electron-ion collision frequency. Different cases have been studied to better understand the role played by the magnetic drift and the electric potential. First case, without electric potential and without magnetic drift (black square), second case without electric potential and with magnetic drift (green plus sign), third case with electric potential and without magnetic drift (blue triangle) and the last case with both electric potential and magnetic drift (red cross)

in the intermediate collisionality. In ${ }^{28}$ and ${ }^{36}$, MTMs are found unstbale is collisionless regime. It appears that the trapped particle in this regime play an important role in the MTMs destabilization. For the set of parameters presented in the Table I we add in GKW code the effect of trapped particles which amounts to adding the following term in the Eq25

$$
I V=+\frac{\mathbf{b}}{m} \cdot\left(\mu \nabla B+\nabla \xi_{\Omega}\right) \frac{\partial f}{\partial v_{\|}}
$$

The term IV 26 calculates the mirror trapping terms. In the Fig 2, when $10^{-3}<v_{e i}^{G K W}<10^{-2}$, the trapped particles destabilize MTMs and when $v_{e i}^{G K W}>10^{-2}$ MTMs are stabilized. At low collisionality, $v_{e i}^{G K W}<$ $10^{-3}$, the trapped electrons modes (TEM) are the dominant modes, blue square curve in the Fig 2 Starting with the Fig 2, is not possible to conclude about the stabilization of MTMs by the trapped particles in collisionless regime. Before adding an additional difficulty, which is the effect of the trapped particles, in the understanding of the MTMs destabilization, let's look at the first time the role played by the magnetic drift. In the next part, a comparison between the analytical model, considering only the effect of magnetic drift (Eq. (16)), with GKW simulation is presented.

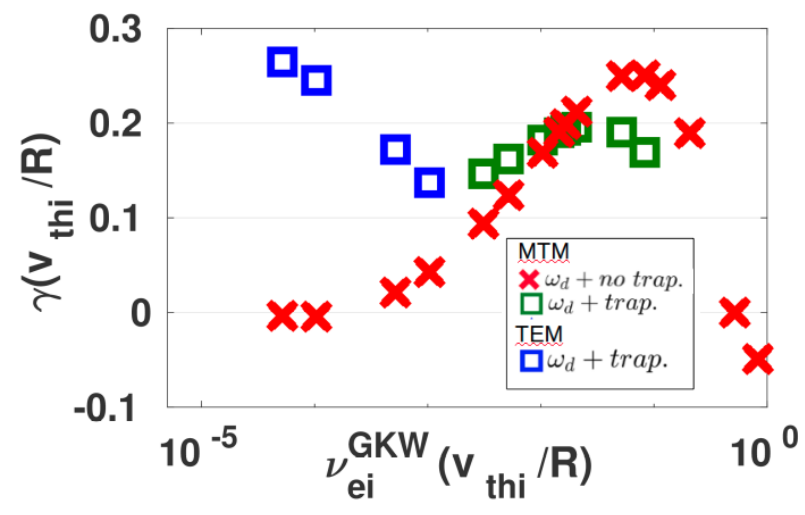

Figure 2: Growth rate of the microtearing mode as function of the electron-ion collision frequency.

Different cases have been studied to better understand the role played by the trapped particles. The red cross curve is the same curve presented in the Fig. 1. the electric potential and magnetic are considered whereas the trapped particles are neglected. The effect of trapped particles is added in the GKW code, blue and green square curves.

\section{B. MTMs destabilized by the Magnetic drift}

Using the set of parameters in Table $\mathrm{I}$ and the numerical resolution in Table II a comparison between Eq.(16) and numerical simulations has been done. Fig. 3 shows the numerical and analytical growth rate of MTMs as a function of the electron-ion collisionality frequency, $v_{e i}^{G K W}$. To compare the mode frequency and growth rate of MTMs with GKW simulations, the theoretical collision frequency, $\hat{v}_{e i}$, has been normalized using GKW normalization: $v_{e i}^{G K W}=\hat{v}_{e i} \frac{k_{\theta} \rho_{i}}{2} \sqrt{\frac{m_{e}}{m_{i}}} \frac{R}{L_{T e}}$.

In Fig. 3, it appears that MTMs are stable at both low and high collision frequency. At intermediate collisionality $v_{e i}^{G K W} R / v_{t h i} \sim 10^{-2}-10^{-1}$, typical the pedestal region, MTMs are destabilized. Analytical theory and simulations are found to agree. Quantitatively, the agreement is satisfactory in the intermediate whereas differences start to arise at low and high collisionalities. At low collisionality, the expansion of the perturbed distribution function over a basis of Legendre polynomials restricted to the two first components to express the collision operator could explain the difference observed between the analytical calculation and numerical simulations. At high collisionality, the current sheet width becomes wider, and the "constant $\psi$ " approximation is debatable ${ }^{11}$. The main result here is that the magnetic drift plays an important destabilizing role but only in conjecture with a finite collisionality. The fair agreement between the linear theory and GKW simulations and the structure of the mode presented in the Fig. 4legitimates a posteriori the use 

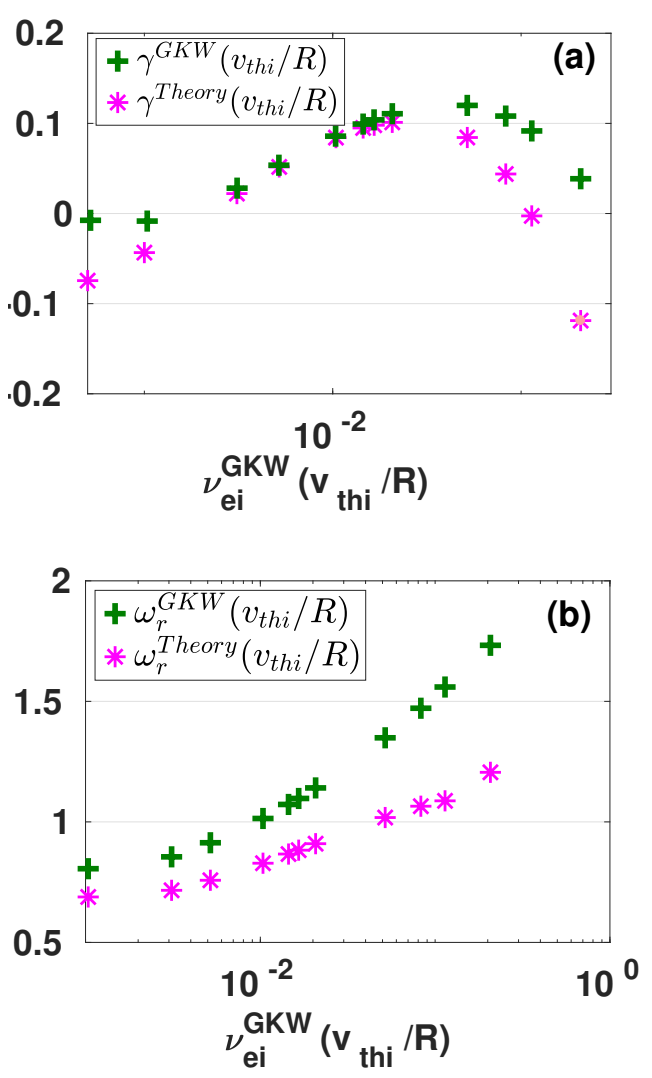

Figure 3: Left: analytical and numerical linear growth rates. Right: the analytical and numerical real frequency of microtearing modes as a function of electron-ion collision frequency $v_{e i}^{\mathrm{GKW}}$ for the set of parameters presented in Table 1 .

of an effective magnetic drift in the electromagnetic model.

\section{Structure of microtearing modes}

In the GKW 32 code, MTMs are clearly identified by their eigenfunctions.

Numerical simulations show that the perturbed electric potential $\hat{\phi}$ is odd and extended along the magnetic field lines whereas the vector potential $\hat{A}_{\|}$is even and more localized around the low field side midplane. This agrees with the classical theory of tearing/microtearing mode. Fig. 4 shows the real (blue curve) and imaginary (red curve) parts of the eigenfunctions for linear simulations. The black curve represents the real part of the mode found analytically using the set of parameters given in TableI In the Fig. 4. (a), the blue and black curve represent the real part of the vector potential $\hat{A}_{\|}(s)$ along the magnetic field line. $\hat{A}_{\|}(s)$ is even and localized around the resonant surface. The radial structure expected by the theory (Eq.
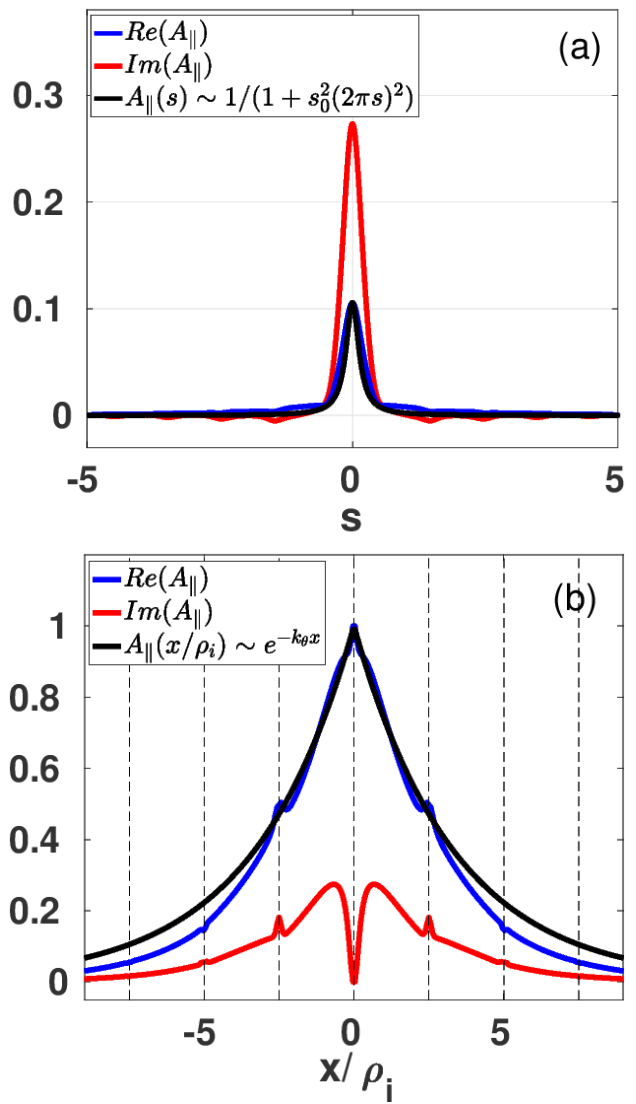

Figure 4: Real and imaginary parts of the numerical eigenfuntion of a MTM at $v_{e i}^{G K W}\left(v_{t h i} / R_{r e f}\right)=0.016$ using the set of parameters given in Table $\mathbb{I}$.

(a): vector potential $\hat{A}_{\|}(s)$ along the magnetic field line in the ballooning space.

(b): radial structure of the vector potential $A_{\|}(x)$ in real space.

Dashed lines indicate resonant surfaces

10 is recovered numerically: $\hat{A}_{\|}(\eta)=\hat{A}_{\|}(0) \frac{1}{1+s_{0}^{2} \eta^{2}}$. The localization of the vector potential near $\eta=0 \mathrm{a}$ posteriori justifies the use of an effective magnetic drift in the analytical calculation. Moreover, in the radial direction, away from the current layer $A_{\|}$decays as $A_{\|}(0) e^{-\left|k_{\theta} x\right|}$ (Fig. 4). (b)).

\section{Electric potential and magnetic drifts destabilize MTMs in conjunction with collisions}

Now, the full electromagnetic calculation is directly compared with GKW simulations. Fig. 5 shows the real and the imaginary part of the electric potential along the magnetic field and in the radial direction.

$\hat{\phi}$ is odd and extended along the magnetic field lines and localized around the resonant surface. In Fig. 6, the linear growth rate of MTMs obtained from the eigenvalue code that solves KRMHD equations is 

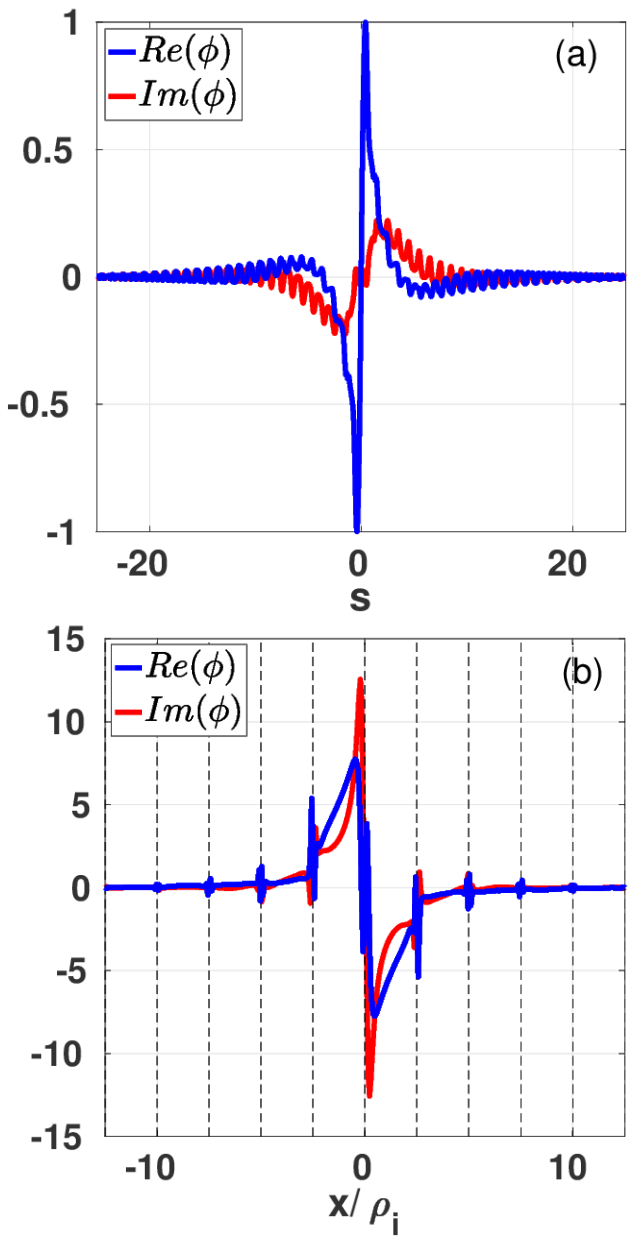

Figure 5: Real and imaginary parts of the numerical electric potential eigenfunction of a MTM at $v_{e i}^{G K W}\left(v_{t h i} / R_{r e f}\right)=0.016$ using the set of parameters given in Table 1 .

(a): electric potential $\hat{\phi}$ along the magnetic field line in the ballooning space.

(b): radial structure of the electric potential $\hat{\phi}(x)$ in the real space.

Dashed lines indicate resonant surfaces.

Table III: Revelant input parameters used for the KRMHD model and the linear simulations with GKW.

\begin{tabular}{cccccccc}
\hline$q$ & $\mathrm{~s}$ & $\beta(\%)$ & $R / L_{T e}$ & $R / L_{T i}$ & $R / L_{n e}$ & $\epsilon$ & $k_{\theta} \rho_{i}$ \\
\hline 2.2 & 0.3 & 0.9 & 10 & 0 & 0.26 & 0.22 & 0.3 \\
\hline
\end{tabular}

compared with the numerical simulations using GKW. A reasonable agreement between "Solve_AP" and the GKW simulations is found. The main result is, MTMs are only destabilized in the presence of collisions. The magnetic drift and the electric potential play and important role in the MTM destabilization that in the local limit and neglecting trapped particles. There is a fair agreement between "Solve_AP" and numerical simulations despite the approximations done, i.e $\psi$ -
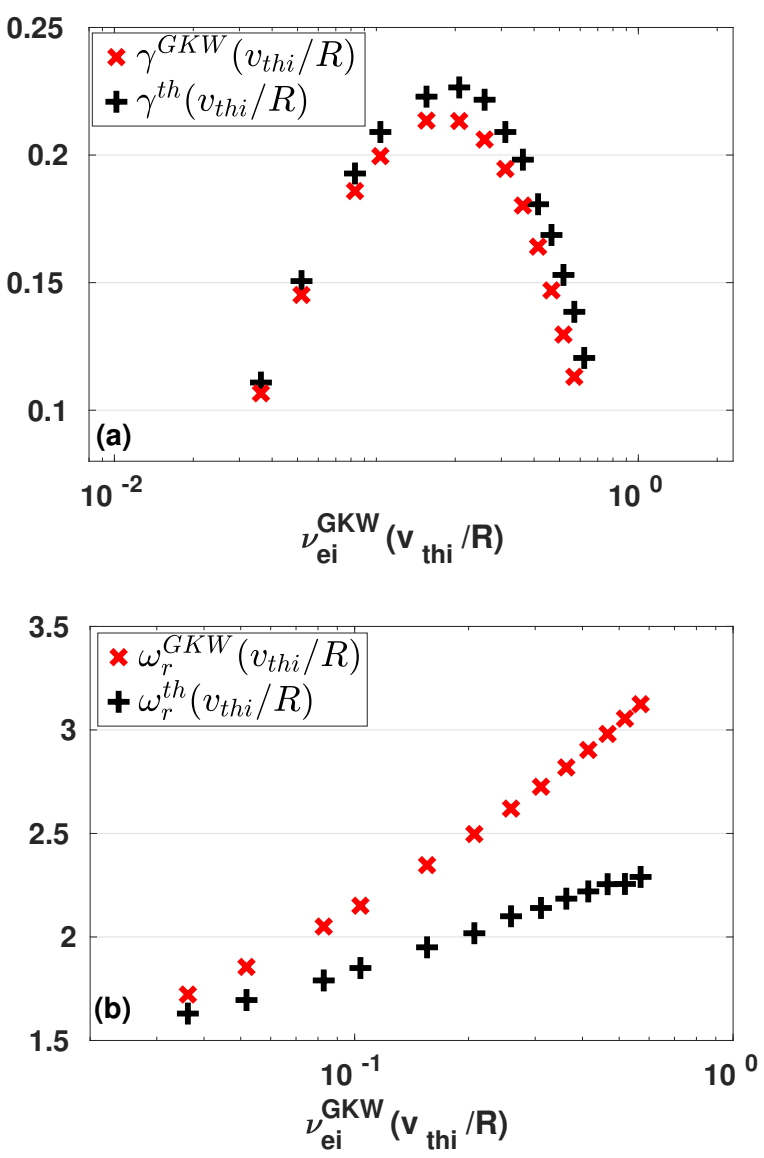

Figure 6: Comparison between linear analytical growth rate (a) and frequency (b) of MTMs with the GKW growth rate of these modes as the function of the electron-ions frequency using the set of parameters presented in Table III.

constant in the current layer and the use of an effective curvature in the KRMHD model

\section{CONCLUSION}

In conclusion, a kinetic dispersion relation of microtearing modes has been developed to model the current sheet including the electric potential, magnetic drift and collisions. It has been compared direclty with numerical simulations using the gyrokinetic code GKW. Electric potential and magnetic drift play an important role in MTM destabilization. The localisation of the vector potential in the ballooning space allows using an effective curvature. Step by step the magnetic drift and electric potential are included in kinetic reduced MHD model and compared with GKW simulations. An analytical dispersion relation including only the magnetic drift is formulated. A fair agreement between the analytical model and GKW simulations 
is observed. It appears that the magnetic drift can destablize MTM only in conjunction with a finite collisionality. Then, the electric potential is added and a reasonable agreement between the numerical simulations and a reduced kinetic MHD model is found. The main result is that MTMs are destabilized by the electric potential and magnetic drift onlyin conjecture with a finite collisionality. Then, we have compared the radial structure of the vector potential assumed by the theory in real and ballooning space with that obtained from numerical simulations. We have found a good qualitative agreement between the two structures. It is very important to stress that the study of the stability of microtearing especially at low collisionality requires a very high resolution to describe correctly the current sheet. The purpose of the future work is to improve the kinetic reduced MHD model by taking into account the physic of the trapped particles to understand their role in the MTMs destabilization.

\section{ACKNOWLEDGMENTS}

The authors thank I. Predebon, C. M. Roach, S. Sareelma, J. C. Hillesheim, L. Frassinetti for fruitful discussions. This work was granted access to the HPC resources of Aix-Marseille University financed by the project Equip@Meso (ANR-10-EQPX-29-01) of the program Investissements d'Avenir supervised by the Agence Nationale pour la Recherche. Part of this work was performed by using computational resources provided by the MARCONI-FUSION HPC.

\section{REFERENCES}

${ }^{1}$ The ASDEX Team, Nucl. Fusion 29 (1980)

${ }^{2}$ F. Wagner, G. Becker, K. Behringer, D. Campbell, A. Eberhagen, W. Engelhardt, G. Fussmann, O. Gehre, J. Gernhardt, G. V. Gierke et al. Phys. Rev. Lett. 49, 1408 (1982)

${ }^{3}$ P.B. Snyder, T.H. Osborne, K.H. Burrell, Phys. Plasmas 19, 056115 (2012).

${ }^{4}$ P. B. Snyder, N. Aiba, M. Beurskens, R. J. Groebner, L. D. Horton, A. E. Hubbard, J. W. Hughes, G. T. A. Huysmans, Y. Kamada, A. Kirk, C.Konz, A. W. Leonard, J. Lonnroth, C. F. Maggi, R. Maingi, T. H. Osborne, N. Oyama, A. Pankin, S. Saarelma, G. Saibene, J. L. Terry, H. Urano, and H. R. Wilson, Nucl. Fusion, 49: 085035, 2009.

${ }^{5}$ D.R. Hatch, M. Kotschenreuther, S.Mahajan, P. Valanju, F. Jenko, D. Told, T. Görler, S. Sareelma, Nucl. Fusion, 56: 104003, 2016.

${ }^{6}$ D.J Applegate, C. M. Roach, J. W. Connor, S. C. Dorland, R. J Hastie and N. Joiner, Phys. Control. Fusion, 49: 1113, 2007.

${ }^{7}$ K. Wong, S. Kaye, D. Mikkelsen, J. Krommes, K. Hill, R. Bell and B. LeBlank, Phys. Rev. Lett, 99: 135003, 2007.

${ }^{8}$ W. Guttenfelder , J. Candy, S.M. Kaye, W.M. Nevins, R.E. Bell et al., Phys. Plasmas, 19: 022506, 2012.

${ }^{9}$ R. D. Hazeltine, D. Dobrott and T.S. Wang, Phys. Fluids, 18:17781786, 1975.
${ }^{10}$ J.F. Drake and Y.C. Lee, Phys. of Fluids, 20: 1341-1353, 1977.

${ }^{11}$ N. T. Gladd, J. F. Drake, C. L. Chang, and C. S. Liu Phys. Fluids 23: 1182,1990

${ }^{12}$ P. J. Catto and M. Rosenbluth, Phys. Fluids, 24: 243-255, 1981.

${ }^{13}$ J.W. Connor, S.C. Cowley and R.J. Hastie, Plasma Phys. Control. Fusion, 32: 799-817 (1990).

${ }^{14}$ A. I Smolyakov, Sov. J. Plasma Phys., 15: 1153-1159, 1989.

${ }^{15}$ X.Garbet, F. Mourgues, A. Samain Plasma Phys. Control. Fusion,30: 343-363, 1988.

${ }^{16}$ X.Garbet, F. Mourgures, A. Samain Plasma Phys. Control. Fusion, 32: $131-140,1990$

${ }^{17}$ A.Zocco, N. F.Loureiro, D. Dickinson, R. Numata and C.M. Roach Plasma Phys. Control. Fusion, 57: 065008, 2015.

${ }^{18}$ S. Nasr, A. I. Smolyakov, P. Migliano, D. Zarzozo, X. Garbet, and S. Benkadda Phys. Plasmas, 25: 074503, 2018.

${ }^{19}$ J.W. Connor, and R.J. Hastie, Physics of Fluids, 1987.

${ }^{20}$ S. Moradi, I. Pusztai, W. Guttenfelder, T. Fulop, and A. Molln, Nucl. Fusion, 53: 063025, 2013.

${ }^{21}$ H. Doerk, F. Jenko, T. GÃúrler, D.Told, M.J. Pueschel, and D.R. Hatch Phys. Plasmas, 19: 055907, 2012.

${ }^{22}$ D. Told, F. Jenko, P. Xanthopoulous, L.D. Horton, E. Wolfrum and ASDEX Upgrade Team, Phys. Plasmas, 15: 102306, 2008.

${ }^{23}$ N. Ohyabu, G. Jahns, R. Stambaugh, and E. Strait, Phys. Rev. Lett. , 58: 120, 1987.

${ }^{24}$ M. Kotschenreuther, W. Dorland, Q.P. Liu, M.C. Zarnstorff, R.L. Miller, Y.R. Lin-Liu, Nucl. Fusion, 40: 677, 2000.

${ }^{25}$ H.R. Wilson, J.-W. Ahn, R.J. Akers, D. Applegate, R.A. Cairns, J.P. Christiansen, J.W. Connor, G. Counsell, A. Dnestrovskij, W.D. Dorland, M.J. Hole, N. Joiner, A. Kirk, P.J. Knight, C.N. LashmoreDavies, K.G. McClements, D.E. McGregor, M.R. OâĂŹBrien, C.M. Roach, S. Tsaun, G.M. Voss, Nucl. Fusion, 44: 917, 2004.

${ }^{26}$ C.M. Roach, D.J. Applegate, J.W. Connor, S.C. Cowley, W.D. Dorland, R.J. Hastie, N. Joiner, S. Saarelma, A.A. Schekochihin, R.J. Akers, C. Brickly, A.R. Field, M. Valovic, and the MAST team, Plasma Phys. Control. Fusion, 47: 233, 2005.

${ }^{27}$ D.R. Smith, W. Guttenfelder, B.P. LeBlanc, D.R. Mikkelsen, Plasma Phys. Control. Fusion, 53: 035013, 2011.

${ }^{28}$ D. Dickinson, C.M. Roach, S. Saarelma, R. Scannell, A. Kirk and H.R. Wilson, Phys. Rev. Let., 108: 135002, 2012.

${ }^{29}$ A.K.Swamy, R.Ganesh, J.Chowdhuri, S.Brunner, J.Vaclavik, and L. Villard, Phys.Plasmas 21: 082513, 2014.

${ }^{30}$ I. Predebon, F. Sattin, M.Veranda Phys. Plasmas, 20: 040701, 2013.

${ }^{31}$ D. Carmody, M.J Pueschel, and P.W Terry Phys. of Plasmas, 20: 052110, 2013.

${ }^{32}$ A.G. Peeters et al., Computer Physics Communication, 180: 2650, 2009.

${ }^{33}$ M.Hamed, M.Muraglia,Y. Camenen, X. Garbet, Contrib. Plasm. Phys., 58: 529-533, 2018

${ }^{34}$ M. Hamed, M. Muraglia, Y. Camenen, X. Garbet, J. Phys.: Conf. Ser. 1125: 012012, 2018

${ }^{35} \mathrm{~J}$. Heading, An introduction to Phase Integral Methods, (Wiley, NY, 1962)

${ }^{36}$ A.K.Swamy, R.Ganesh, J.Chowdhuri, S.Brunner, J.Vaclavik, and L. Villard, Phys.Plasmas 21: 082513, 2014.

${ }^{37}$ A. Poyé, O. Agullo, A. Smolyakov, S. Benkadda and X. Garbet, Phys. Plasmas 20: 020702, 2013.

${ }^{38}$ L. Spitzer, Physics of Fully Ionized Gases, Interscience, 1956

${ }^{39}$ A.G. Peeters, R. Buchholz, Y. Camenen, F.J. Casson, S. Grosshauser, W.A. Hornsby, P. Manas, P. Migliano, M. Siccinio, A.P. Snodin, D. Strintzi, T. Sung, G. Szepesi, D. Zarzoso, "GKW how and why", manual, 2007-2015, http:/ /www.gkw.org.uk/tikiwiki/Manual

${ }^{40}$ K. Hallatschek Phys. Rev. Lett., 95: 0550022005. 